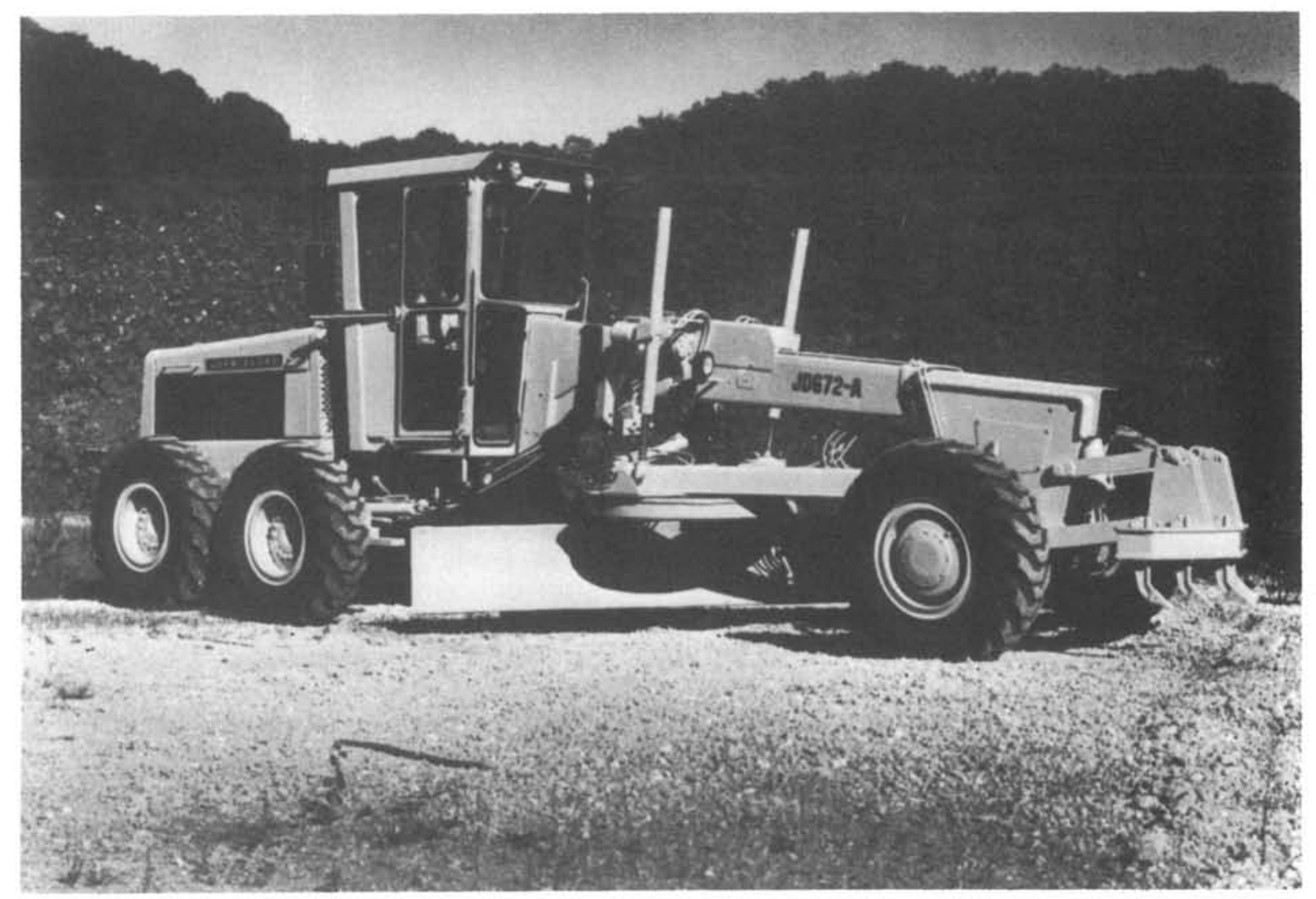

Figura 1.-Motoniveladora JD672 A con tracción a las ruedas delanteras.

\title{
aplicación de un sistema de transmisión auxiliar al equipo de movimiento de tierras para aumentar su productividad
}

John I. Henn

480-1

\begin{abstract}
sinopsis
La productividad del equipo industrial móvil se puede aumentar aplicando un sistema de transmisión auxiliar a las ruedas no motrices, sobre todo cuando las condiciones de tracción son limitadas o variables. A fin de aumentar el esfuerzo de tracción y la estabilidad direccional se ha aplicado este sistema a las ruedas delanteras de las motoniveladoras John Deere, con el uso de accionamiento hidráulico y control electrónico. Este sistema podria aplicarse a otros tipos de equipo industrial móvil.
\end{abstract}

La limitación de la tracción y del control de la dirección son dos parámetros importantes que afectan a la productividad de las motoniveladoras. La capacidad de transmisión en las ruedas delanteras puede aumentar esta productividad, sobre todo en condiciones de tracción limitada.

La finalidad de este artículo es la de exponer el desarrollo de un sistema de transmisión para las ruedas delanteras de las motoniveladoras John Deere, accionado hidráulicamente y controlado electrónicamente. 
Cuando se estudia y se aprueba el concepto de ruedas delanteras motrices en una motoniveladora se observan varias ventajas:

En primer lugar, las motoniveladoras suelen perder tracción en las marchas cortas. En este caso, la tracción en las ruedas delanteras aumenta el esfuerzo de tracción, con lo que mejora la productividad. La máquina puede moverse por los sitios más difíciles sin perder la tracción ni alterar una superficie que acaba de ser nivelada.

Las motoniveladoras se mueven frecuentemente en superficies que dificultan la tracción, como arena, barro, hielo y nieve. Aunque estas condiciones adversas no suelen durar mucho, con el fin de mantener la productividad a un nivel razonable e impedir que la máquina quede inmovilizada, se necesita un esfuerzo de tracción apropiado.

En segundo lugar, dado que la hoja de una motoniveladora suele situarse en ángulo durante los trabajos de nivelación, es preciso contrarrestar la fuerza lateral para mantener un control adecuado de la máquina. Aunque el peso adicional sobre el eje delantero y la capacidad de inclinación de las ruedas representan una ayuda, la transmisión en las ruedas delanteras es la mejor solución para mejorar el control direccional (figura 2), especialmente importante cuando se trabaja en curvas o lugares estrechos, donde las cargas laterales no se encuentran alineadas con las ruedas de tracción principal.

Las motoniveladoras de chasis articulado, más versátiles, pueden trabajar en posición articulada, en la que las cargas laterales no están alineadas con las ruedas de tracción principal, aumentándose aún más la fuerza lateral. En estas condiciones, la tracción en las ruedas delanteras es importante para poder mantener el control de la dirección (figura 3).

Cuando se planteó la selección de un sistema de tracción a las ruedas delanteras para motoniveladoras, se idəntificaron y estudiaron una serie de parámetros de diseño:

1. Aumentar el rendimiento o productividad de la máquina, especialmente en condiciones de tracción limitada o variable.

2. Mantener la capacidad actual de dirección, que incluye:

- Un radio mínimo de giro de $6,85 \mathrm{~m}$.

- Una capacidad de inclinación de $20^{\circ}$ de las ruedas delanteras.

3. Mantener en el mínimo posible los cambios en el diseño actual de las motoniveladoras y proporcionar la opción de fabricarlas con o sin transmisión delantera.

4. Asegurar la compatibilidad con las caracteristicas de cambio de marchas del sistema de tracción principal.

5. Dar la posibilidad de acoplar y desacoplar la transmisión auxiliar desde el puesto del operador.

6. Impedir las sobrecargas de par en las ruedas delanteras.

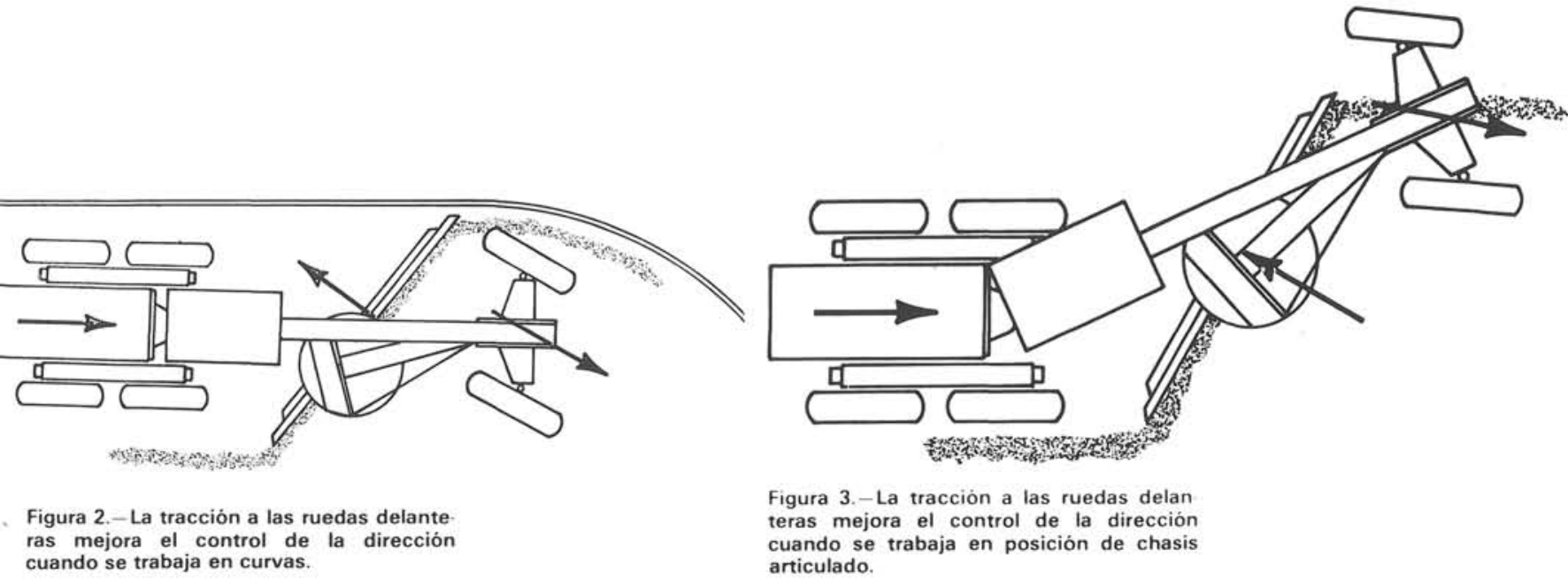


7. Proporcionar una acción diferencial de blocaje en las ruedas delanteras.

8. Unificar los componentes de la transmisión delantera de todos los modelos de motoniveladoras y otros productos industriales.

Después de estudiar cuidadosamente los diversos conceptos para el diseño, se decidió que el sistema hidráulico era el que mejor podia responder a los parámetros que nos habíamos fijado.

Se evaluó las posibilidades de un sistema de control compensador de la presión constante. El control era relativa. mente sencillo y económico. El sistema proporcionaria a las ruedas delanteras un par de tracción constante en buenas condiciones de tracción; pero si estas condiciones empeoraban o se reducía el peso sobre el eje delantero al recibir la hoja una presión hacia abajo, el rendimiento del sistema se hacía inaceptable.

Se decidió que, a fin de mantener un rendimiento óptimo en todas las condiciones, era preciso controlar la velocidad de las ruedas delanteras con relación a la de las traseras. Esto se consiguió con un control electrónico que incorporaba un sistema de control de realimentación en circuito cerrado. Este concepto de regulación de la velocidad se desarrolló hasta cristalizar en el diseño actual, que proporciona la tracción delantera a las motoniveladoras John Deere.

El sistema de transmisión incluye tres subsistemas combinados funcionalmente:

- Un sistema hidráulico de transmisión para proporcionar potencia a las ruedas delanteras.

- Un sistema electrónico de control para mantener constante y precisa la velocidad de los motores de las ruedas delanteras.

- Un sistema de acoplamiento a las ruedas delanteras que proporciona una conexión sueve y correcta respecto a la tranśmisión principal.

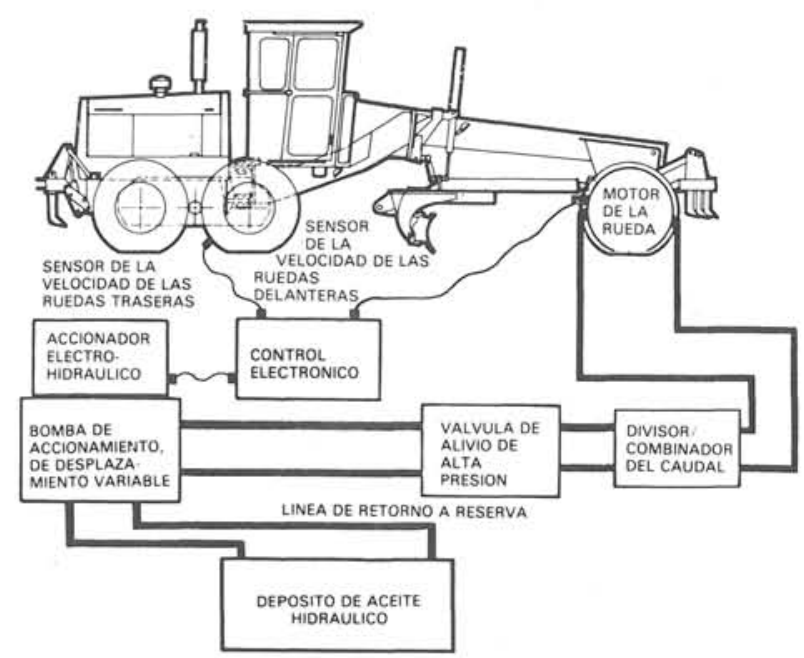

Figura 4.-Esquema de los componentes de la ruedas delanteras de la motoniveladora.

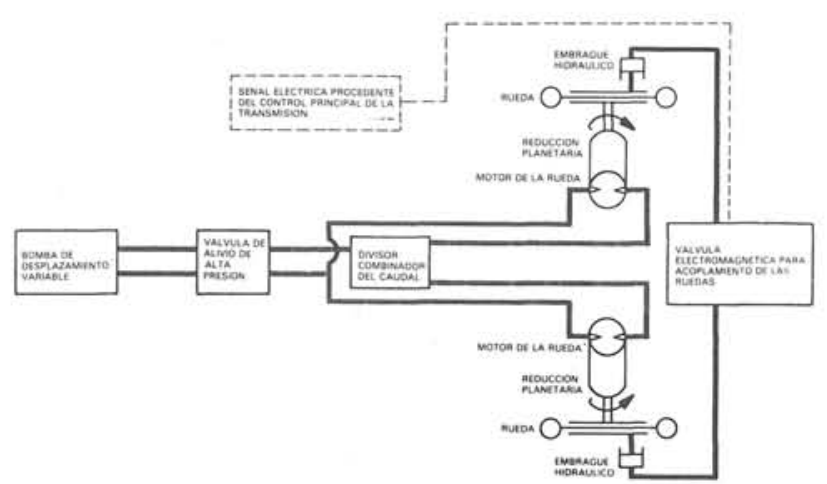

Figura 5.-Esquema de los componentes del accionamiento hidráulico y del acopla miento de las ruedas del sistema de tracción delantera de la motoniveladora.

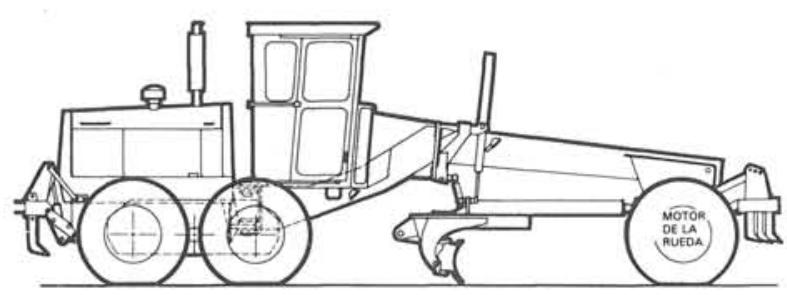

Fig. 6.- Las ruedas delanteras giran a velo cidad superior a la de sus motores cuando no existe patinaje.

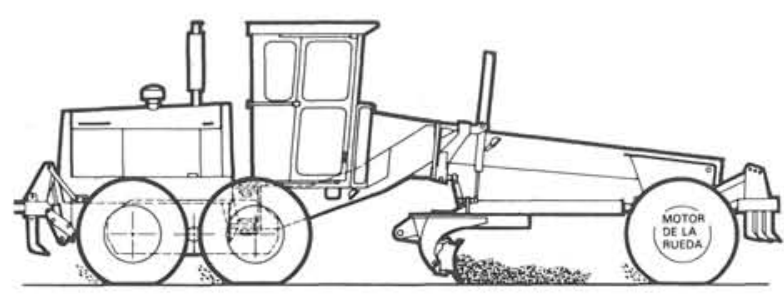

Figura 7.-Las ruedas delanteras se acoplan a sus motores y desarrollan el par de tracción cuando patinan las traseras. 


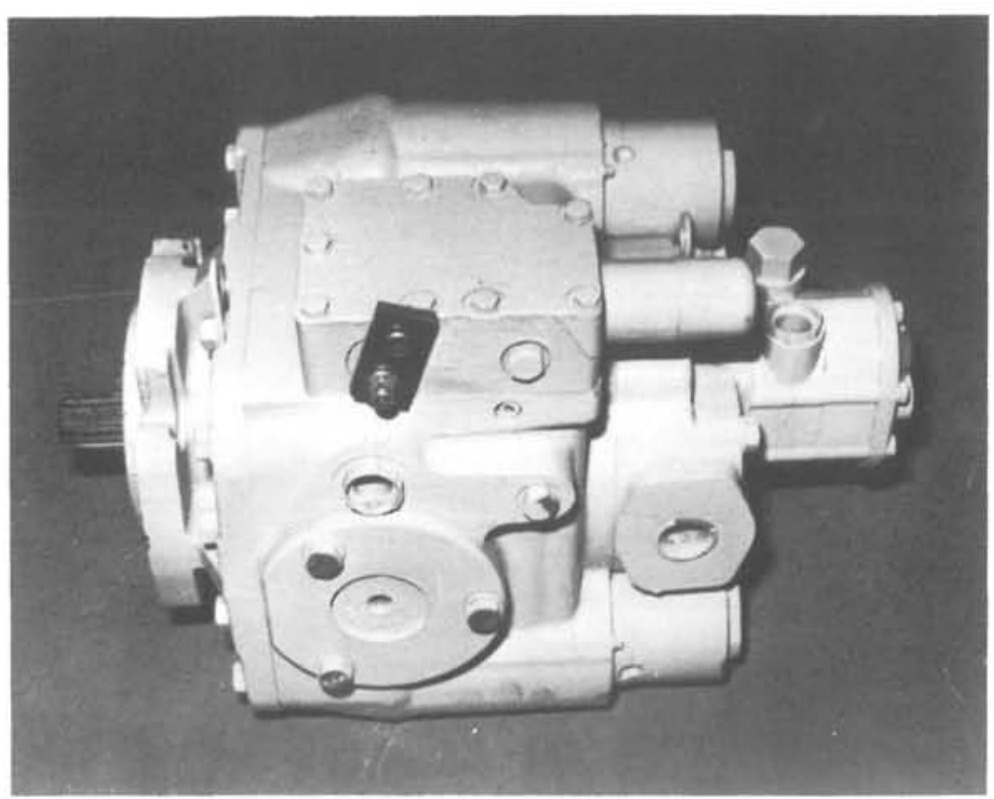

Figura 8.-Bomba de accionamiento.

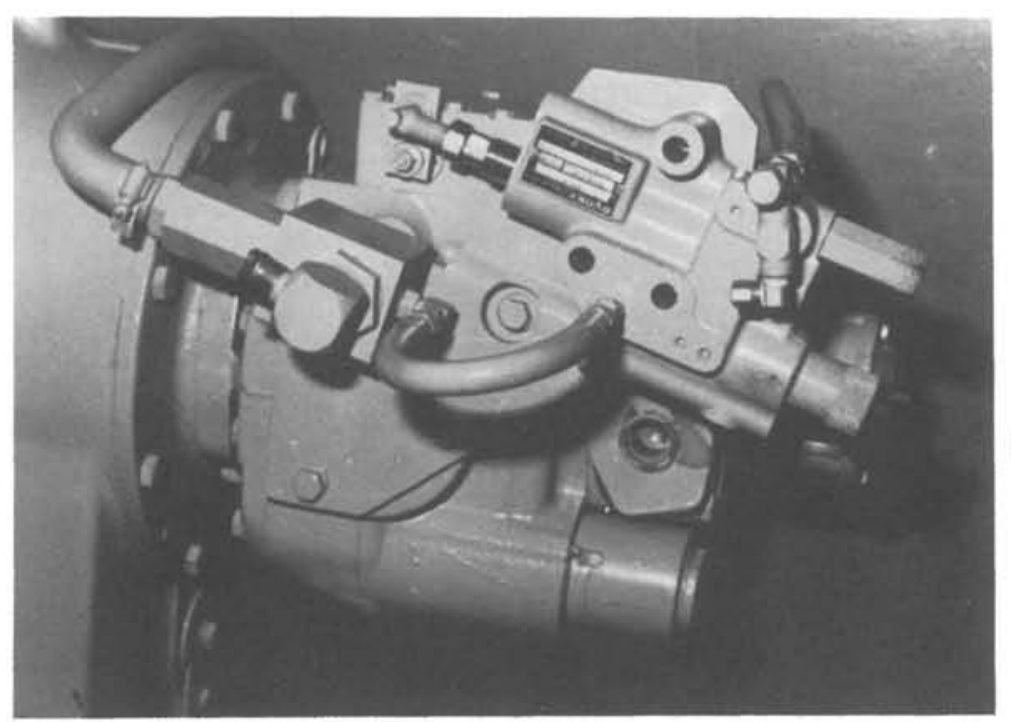

Figura 10.-Accionador montado en la bomba.
El sistema hidráulico de transmisión incluye una bomba de accionamiento reversible y desplazamiento variable, montada en la parte posterior del motor y que proporciona aceite hidráulico a los dos motores hidráulicos de las ruedas, de desplazamiento fijo, montados en las ruedas delanteras (figura 4 ). Incorpora una válvula de alivio de alta presión para proteger el sistema contra las sobrecargas de par así como un divisor/combinador de la alimentación, que proporciona un blocaje diferencial parcial de las ruedas delanteras.

El sistema electrónico de control detecta la velocidad de las ruedas traseras y envia la señal a un accionador electro-hidráulico que regula el desplazamiento de la bomba de accionamiento, a fin de mantener en los motores de las ruedas delanteras una determinada velocidad respecto a la de las ruedas traseras. Un sensor de velocidad, situado en las ruedas delanteras, proporciona la señal de realimentación al circuito cerrado que mantiene la adecuada precisión en la velocidad de las delanteras.

Unos embragues hidráulicos, situados en los conjuntos de rueda, se encargan del acoplamiento entre las ruedas y sus motores.

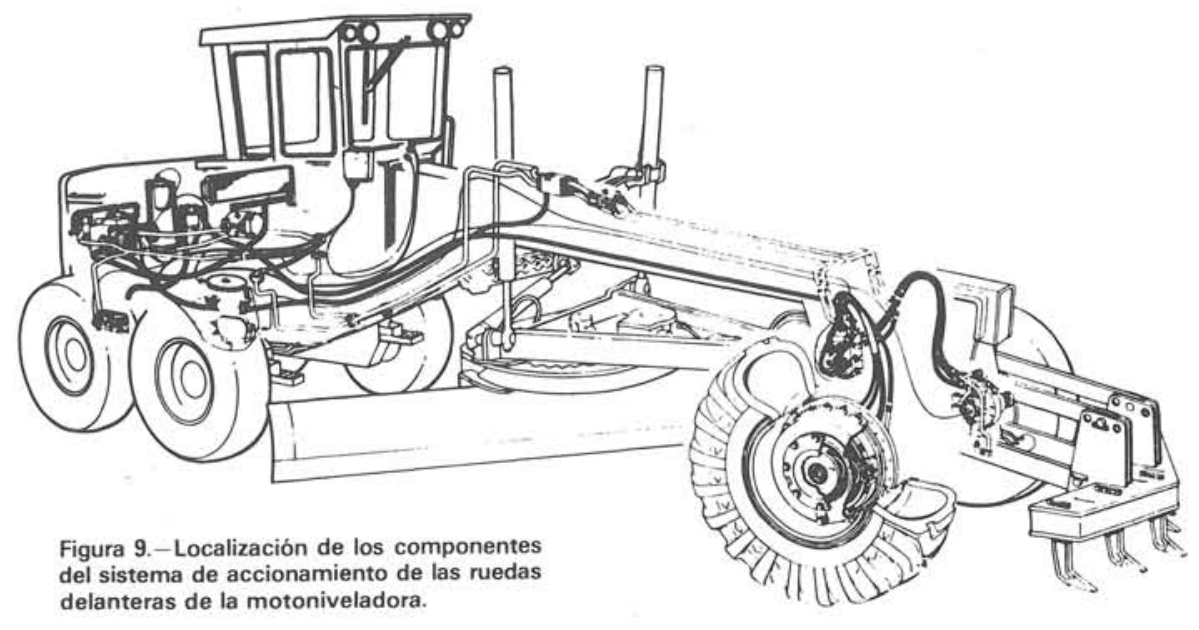




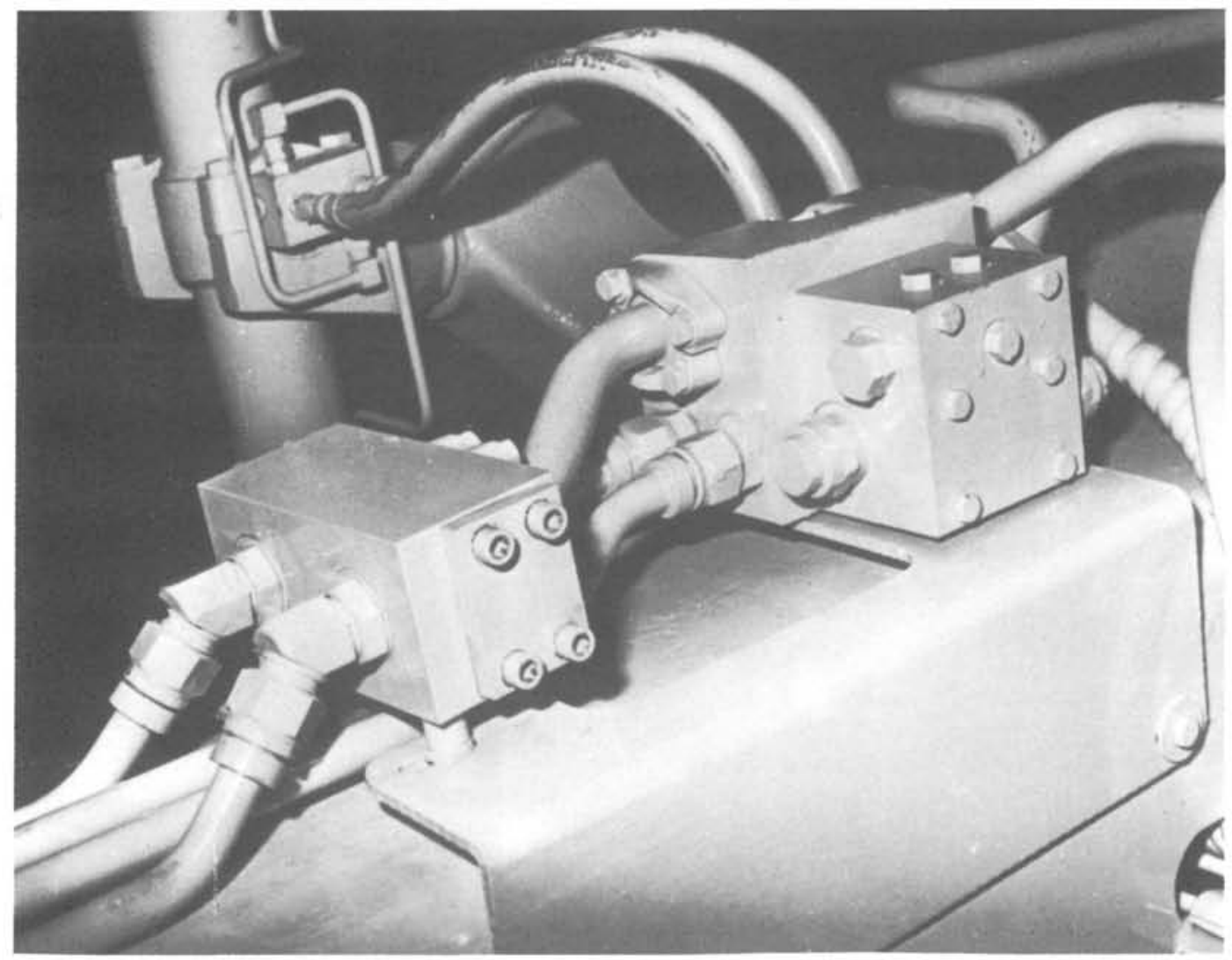

Figura 11.-Válvula de alivio de alta pre sión y divisor/combinador de caudal

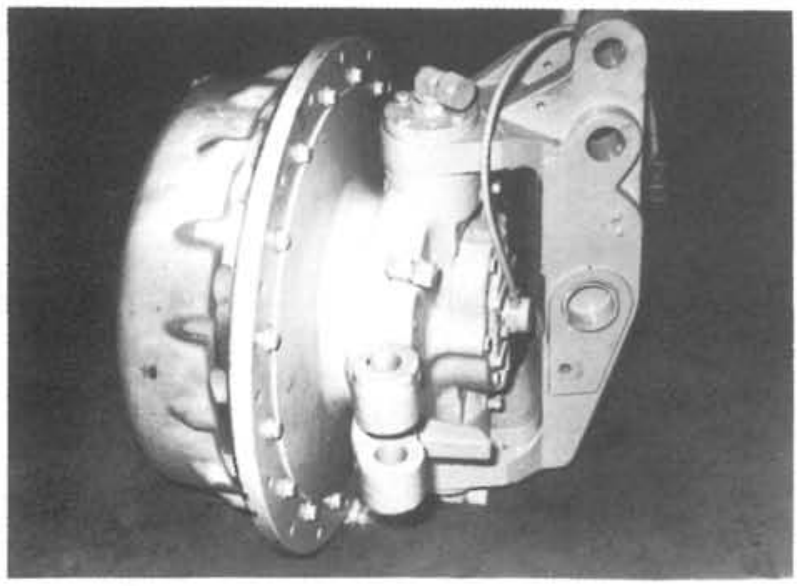

Figura 12.-Motor de la rueda con sensor delantero.

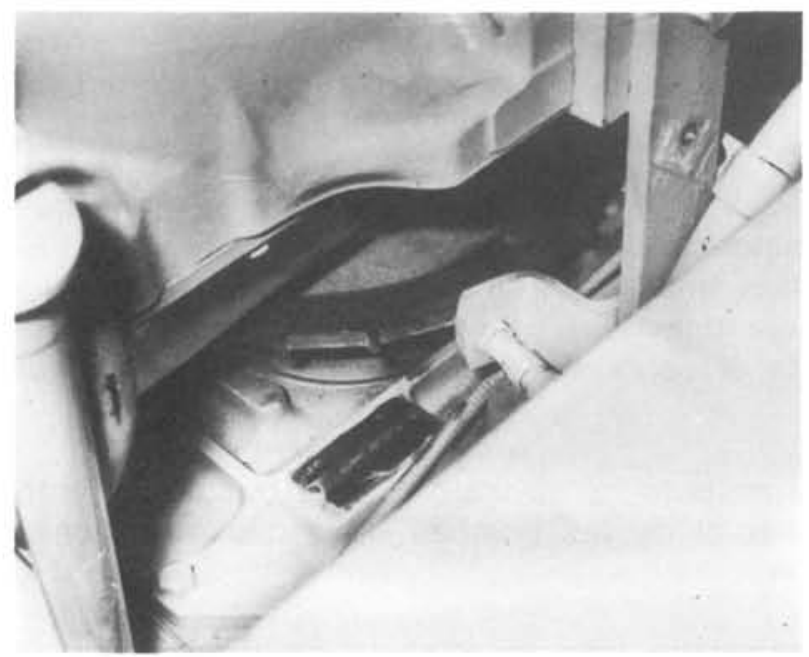

Figura 13.-Sensor de la velocidad de las ruedas traseras.
Una válvula electromagnética envía aceite a presión a los embragues (figuras 5 y 9 ). En el control de la transmisión principal se encuentran unos interruptores para controlar el acoplamiento de las ruedas delanteras, con respecto a la transmisión principal. Este sistema de acoplamiento facilita también la liberación de las ruedas cuando se desconecta el sistema de transmisión o se cambia a velocidades más largas. El sistema deja también libres las ruedas delanteras cuando se cambia o se pone en punto muerto la transmisión principal.

Los embragues hidráulicos actúan también como embragues de sobremarcha cuando la máquina trabaja sin esfuerzo. Este efecto de sobremarcha ocurre porque los motores de las ruedas delanteras están controlados para que se muevan más lentamente que las traseras; como consecuencia, los motores de las ruedas delanteras giran a menor velocidad que ellas (figura 6). 


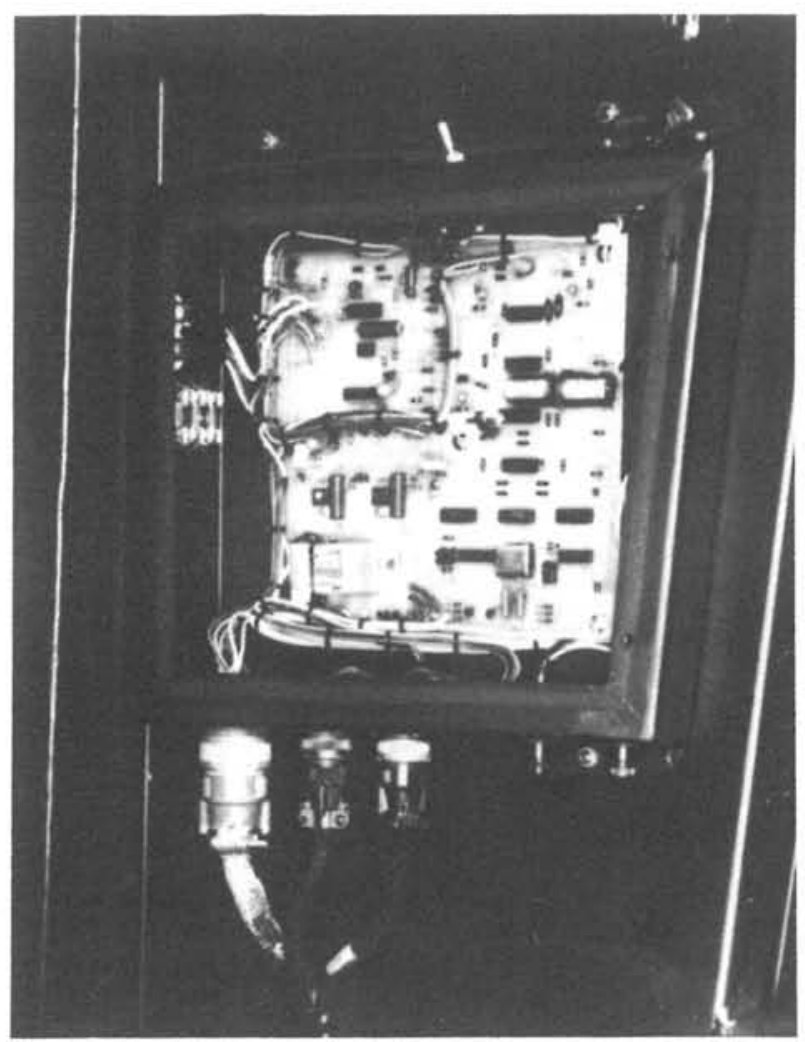

Figura 14.-Caja de control abierta.

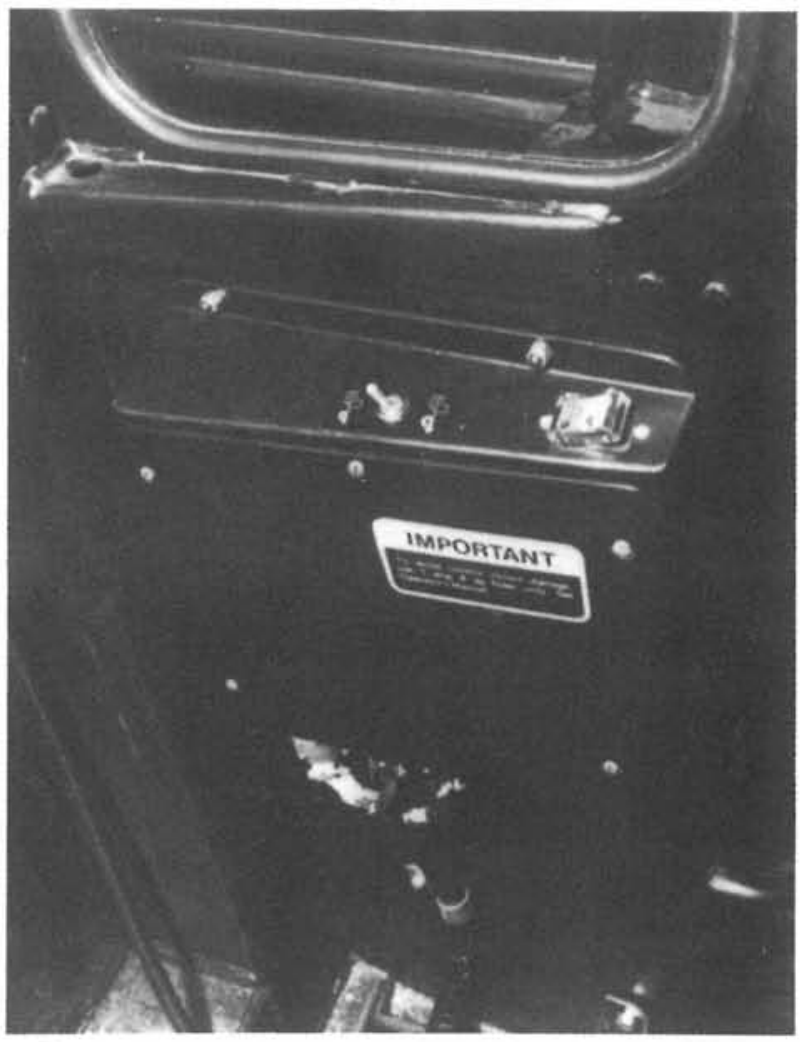

Figura 15.-Caja de control mostrando los controles del operador.

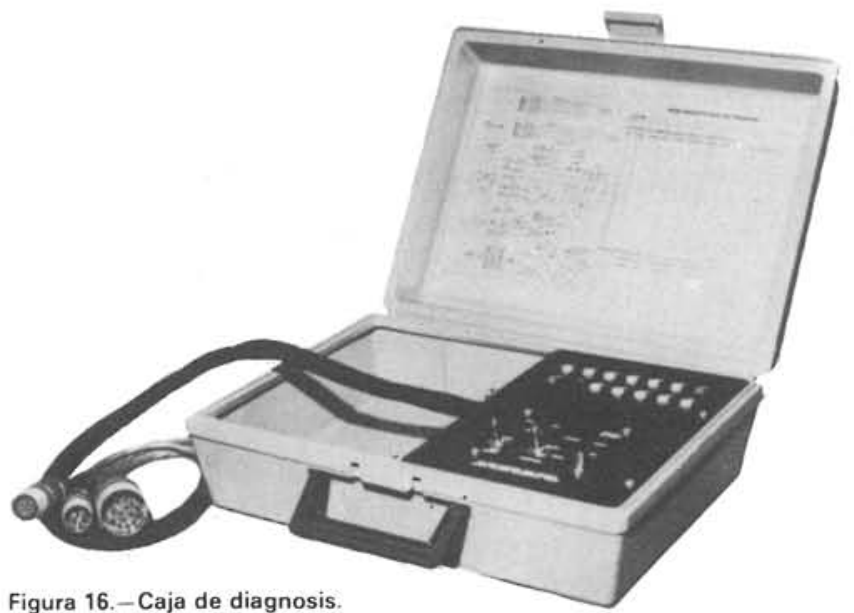

Al acoplar la herramienta de trabajo, la resistencia aumenta y las ruedas traseras patinan en el suelo, con lo cual las delanteras no van ya a más velocidad que sus motores y la presión en la transmisión aumenta a medida que sube la resistencia del par en las ruedas delanteras (figuras 5 y 7). Esta capacidad de sobremarcha impide interferencias de par entre los sistemas de tracción delantero y trasero, sin perjudicar por ello a la sensibilidad de todo el sistema.

Como quiera que el sistema de transmisión incluye un control de la velocidad, se tiene que la presión en la transmisión varia según la resistencia del par de las ruedas delanteras. EI par sólo se desarrolla cuando patinan las ruedas traseras y su magnitud varia según las condiciones de tracción y el peso sobre el eje delantero, por lo que la presión en la transmisión varia desde un valor mínimo hasta el calibrado para que entre en funcionamiento la válvula de alivio de alta presión.

Como se ha dicho antes, la bomba de accionamiento es reversible y de desplazamiento variable. Tiene un diseño de pistón axil, similar al de las bombas utilizadas en muchas aplicaciones de transmisión a presiones elevadas.

El depósito hidráulico proporciona el aceite necesario para la transmisión de las ruedas delanteras, con filtros que protegen el sistema contra la contaminación (figura 9). 
El accionador electro-hidráulico es el elemento de unión entre el sistema electrónico de control y el sistema hidráulico de tracción. El accionador recibe la señal de control, procedente de la caja de control, y varia el desplazamiento de la bomba de accionamiento, proporcionando asi a los motores de las ruedas delanteras la cantidad adecuada de aceite (figuras 4,9 y 10 ).

La válvula de alivio limita las presiones en la transmisión, para impedir que los componentes sufran daños en caso de que patinen las ruedas traseras, y las delanteras tengan la máxima tracción (figuras 4, 9 y 11). Esta capacidad de limitación del par es especialmente importante cuando se utilizan equipos pesados montados en la parte delantera, tales como el arado quitanieves.

El divisor/combinador de alimentación facilita el óptimo rendimiento de la tracción delantera, limitando la alimentación cuando la tracción en las mismas es mínima. Gracias a las características de esta válvula, el dispositivo actúa tanto en marcha adelante como en marcha atrás (figuras 4,9 y 11 ).

El diseño de los motores de las ruedas y de la horquilla de montaje proporciona las caracteristicas de direccionabilidad deseadas.

Se incluyen:

- horquillas de montaje basculantes;

- conductos de distribución del aceite a través de los pivotes de la dirección;

- motores hidráulicos de desplazamiento fijo;

- reducciones por engranajes planetarios dobles;

- embragues hidráulicos que acoplan las ruedas a sus motores.

La velocidad de las ruedas traseras es detectada por un sensor electrónico que cuenta los dientes de un disco especial de freno, situado en la parte superior de la carcasa de la transmisión. El disco va acoplado directamente a la transmisión de las ruedas posteriores (figuras 4,9 y 13 ).

Todos los dispositivos electrónicos van conectados a la caja de control, situada en el puesto del operador. Esta caja de control contiene los circuitos eléctricos que regulan todo el sistema, asi como los controles del sistema de transmisión a las ruedas delanteras (figuras 4,9 y 12 ).

El sistema de transmisión puede ser acoplado y desacoplado por medio de un interruptor situado en la caja de control. El sistema de transmisión se aplica en las marchas $1 .^{a}$ a $4 .^{a}$, tanto adelante como atrás, proporcionando transmisión en todas las marchas de trabajo, al tiempo que se desconecta automáticamente en las marchas más largas. A velocidades superiores no se necesita transmisión delantera porque la potencia de la unidad es limitada (figura 15).

El operador puede elegir también un modo "agresivo", con sobrevelocidad (figura 15). En este modo de funcionamiento, los motores de las ruedas delanteras se regulan de forma que actúen más rápidamente que las ruedas traseras, proporcionándose así capacidad de tracción a las ruedas delanteras aunque no patinen las ruedas traseras. Esta característica es especialmente interesante para mantener el control de la dirección de las ruedas delanteras cuando las traseras no están alineadas con la carga lateral, y es muy conveniente en usos, tales como la limpieza de zanjas, la nivelación en pendientes o en posiciones de chasis articulado (figura 3 ).

El uso de los controles electrónicos presenta un problema nuevo por lo que al servicio se refiere. Los circuitos de control son bastante complejos y se necesitaría un equipo costoso y personal especializado para su servicio. Asi pues, se ha desarrollado una caja de diagnosis que sirva de ayuda al mecánico de servicio (figura 16). Con esta caja y un voltiomimetro, el personal de servicio puede determinar si existe una averia en la caja de control, sin necesidad de verificar cada uno de los circuitos. 
El servicio de la caja de control se presta de acuerdo con un programa de intercambio de componentes. La pieza averiada se devuelve al proveedor, efectuándose un abono al cliente, quien puede entonces comprar un componente a precio reducido. Con piezas caras o complejas, esta técnica de servicio presenta varias ventajas:

- Reduce los costes de servicio del cliente para piezas relativamente costosas.

- Elimina la compra de aparatos de comprobación caros y el entrenamiento técnico, a nivel de concesionario, para la reparación de piezas sofisticadas.

- Reduce la destrucción de piezas de valor elevado. En vez de tirar las piezas, se devuelven a fábrica, donde las mismas personas que las han fabricado son las que las diagnostican y reparan. Las piezas reparadas, con garantía, se devuelven entonces a los concesionarios.

- Como quiera que las piezas averiadas se devuelven al fabricante para su reparación, este último dispone, de inmediato, de la información necesaria para mejorar su diseño. Las mejoras o cambios se pueden incorporar ya a los conjuntos que se devuelven.

Dos de nuestros objetivos primordiales fueron los de conseguir un excelente rendimiento e introducir el menor número posible de cambios en un diseño ya existente. Creemos que el control electrónico ha ayudado a alcanzarlos. Pensamos igualmente que la electrónica proporciona la flexibilidad de diseño necesaria para cambiar de forma económica las características operativas y poner fácilmente a disposición varios modos de operación.

\section{RESUMEN}

1. La productividad del equipo industrial móvil puede aumentarse aplicando un sistema de transmisión auxiliar a las ruedas no motrices, especialmente cuando las condiciones de tracción son limitadas o variables.

2. El sistema de transmisión hidráulica proporciona una máxima flexibilidad de diseño, con cambios mínimos en los modelos ya existentes.

3. Los componentes hidráulicos, de concepción convencional, protegen contra las sobre-

- cargas e incorporan al sistema de tracción la posibilidad de blocaje del diferencial.

4. Para conseguir el máximo rendimiento de un sistema de transmisión auxiliar, en todas las condiciones de tracción, se necesita un sistema de velocidad controlada.

5. Los controles electrónicos permiten varios modos de operación y son muy convenientes para mantener el rendimiento óptimo de la unidad en muchas y muy variadas condiciones de servicio.

6. Los controles electrónicos son relativamente nuevos en usos industriales de unidades móviles y necesitan técnicas de servicio diferentes.

7. El concepto de sistema movido hidráulicamente y controlado electrónicamente puede proporcionar una capacidad de transmisión auxiliar opcional a diversos tipos de equipos móviles que utilizan un elevado número de piezas comunes.

\section{résumé}

APPLICATION D'UN SYSTEME DE TRANSMISSION AUXILIAIRE AUX ENGINS DE TERRASSEMENT POUR AUGMENTER LEUR PRODUCTIVITE John I. Henn

La productivité de l'équipement industriel mobile peut être augmentée par l'application d'un système de transmission auxiliaire aux roues non motrices, surtout quand les conditions de traction sont limitées ou variables. Afin d'augmenter l'effort de traction et Stabilité directionnelle ce système a ete appliqué aux roues avant des motor-graders John Deere, à l'aide d'une commande hydraulique et d'un contrôle électronique. Ce systéme pourrait être appliqué à d'autres types d'equipement industriel mobile.

\section{summary}

APPLYING AN AUXILIARY TRANS. MISSION SYSTEM ON THE LAND MOVEMENT EQUIPMENT TO INCREASE ITS PRODUCTIVITY

John I. Henn

The productivity of the mobile industrial equipment can be increased applying an auxiliary transmission system on the nondrive wheels, especially when the traction conditions are limited or variable. To increase the traction effort and steering stability this system has been applied on the front wheels of the John Deere power graders, using hydraulic drive and electronic control. This system could be applied on other mobile industrial equipment types.

\section{zusammenfassung}

ANBRINGUNG EINES HILFSUEBER TRAGUNSSYSTEMS AN MASCHINEN FUER ERDARBEITEN ZUR ERHOEHUNG DER PRODUKTIVITAET John I. Henn

Die Produktivität von fahrbaren Industrie maschinen kann durch Anbringung eines Hilfsübertragungssystems an nicht angetriebenen Rädern erhöht werden, vor allem bei beschränkten oder veränderlichen Zugverhältnissen. Zur Ehöhung der Zugkraft un Lenkungsstabilität wurde dieses System an die Vorderräder der John-Deere-Planiermas chinen unter Einsatz eines hydraulischen Antriebes und einer elektronischen Kontrolle angebracht. Dieses System könnte für andere Arten von fahrbaren Industriemaschinen eingesetzt werden. 